PROCEEDINGS OF THE

AMERICAN MATHEMATICAL SOCIETY

Volume 126, Number 7, July 1998, Pages 1945-1947

S $0002-9939(98) 04297-\mathrm{X}$

\title{
A NOTE ON THE REDUCIBILITY OF AUTOMORPHISMS OF THE KLEIN CURVE AND THE $\eta$-INVARIANT OF MAPPING TORI
}

\author{
TAKAYUKI MORIFUJI
}

(Communicated by Ronald A. Fintushel)

\begin{abstract}
We give a characterization for the reducibility of automorphisms of the genus 3 Klein curve in terms of the $\eta$-invariant of finite order mapping tori.
\end{abstract}

\section{INTRODUCTION}

The automorphism group of the Klein curve $S$ of genus 3, defined by the equation

$$
x^{3} y+y^{3} z+z^{3} x=0,
$$

is known to be isomorphic to $G=P S L_{2}\left(F_{7}\right)$ [3]. This is a finite group of order 168 and has the presentation

$$
\left\langle s, t \mid s^{2}=t^{3}=(s t)^{7}=[s, t]^{4}=1\right\rangle,
$$

where $[s, t]$ denotes the commutator of $s$ and $t$. Then $G$ can be embedded in the mapping class group $\mathcal{M}_{3}$ of genus 3 . This is a Hurwitz group acting on an oriented closed surface of genus 3 and hereafter we shall consider $S$ as a smooth 2-manifold.

On the other hand, for an element $\varphi$ of $G$ we can construct the mapping torus $M_{\varphi}$ corresponding to $\varphi$. We now endow $M_{\varphi}$ with the metric which is induced from the product of the standard metric on $S^{1}$ and the metric of $S$ such that $\varphi$ acts on $S$ as an isometry. Then the reducibility of the automorphism $\varphi$ (see section 3 for the definition) is characterized via a spectral invariant of $M_{\varphi}$.

Theorem 1. An automorphism $\varphi$ of the Klein curve is reducible if and only if the $\eta$-invariant of the signature operator on mapping torus $M_{\varphi}$ vanishes.

From our earlier work [8], the $\eta$-invariant mentioned above is determined by the action of automorphisms on the first integral homology group $H=H_{1}(S ; \mathbf{Z})$. More precisely, it is described via Atiyah's canonical 2-cocycle [1]. This cocycle is equal to Meyer's signature cocycle [7] up to scalar multiple, so that to prove Theorem 1 we really compute the latter one for each conjugacy class of $G$. The proof of Theorem 1 is given in section 3.

Received by the editors October 15, 1996 and, in revised form, December 20, 1996.

1991 Mathematics Subject Classification. Primary 20F05, 57R20; Secondary 57M10, 57S25.

Key words and phrases. Klein curve, reducibility, $\eta$-invariant, mapping class group. 
TABLE 1. Conjugacy classes of $P S L_{2}\left(F_{7}\right)$

\begin{tabular}{c|c|c|c|c|c|c}
\hline Class $g$ & 1 & 2 & 3 & 4 & $7_{1}$ & $7_{2}$ \\
\hline$|C l(g)|$ & 1 & 21 & 56 & 42 & 24 & 24 \\
\hline
\end{tabular}

\section{2. $P S L_{2}\left(F_{7}\right)$, SignATURE COCYClE AND $\eta$-INVARIANT}

Let $s=\left(\begin{array}{cc}0 & -1 \\ 1 & 0\end{array}\right), t=\left(\begin{array}{cc}0 & -1 \\ 1 & 1\end{array}\right)$ be the generators of $P S L_{2}\left(F_{7}\right)$. It is easy to verify that $s^{2}=t^{3}=(s t)^{7}=[s, t]^{4}=1$. The conjugacy classes of $G$ are well-known (Table 1, see also [4]). In Table 1, each conjugacy class is denoted by the order of its elements. Here the subscripts mean the different classes.

Now we review the definition of Meyer's signature cocycle briefly. See [7] for more detail. For $A, B \in \operatorname{Sp}(2 h ; \mathbf{Z})$, let $V_{A, B}=\left\{(u, v) \in H \oplus H \mid\left(A^{-1}-E\right) u+\right.$ $(B-E) v=0\}$, where $E$ is the identity matrix. Then we define a pairing map $\langle\rangle:, V_{A, B} \times V_{A, B} \rightarrow \mathbf{Z}$ by $\left\langle\left(u_{1}, v_{1}\right),\left(u_{2}, v_{2}\right)\right\rangle=\left(u_{1}+v_{1}\right) \cdot(E-B) v_{2}$, where $\cdot$ is the intersection number. It is easy to see that $\langle$,$\rangle is a symmetric bilinear form on$ $V_{A, B}$. We define $\tau_{h}(A, B)$ to be the signature of $\left(V_{A, B},\langle\rangle,\right)$. This is determined for conjugacy classes.

We choose a symplectic basis $\alpha_{i}, \beta_{i}(i=1,2,3)$ of $S$ as usual. Let $\rho: \mathcal{M}_{3} \rightarrow$ $\operatorname{Sp}(6 ; \mathbf{Z})$ be the epimorphism induced by the action of $\mathcal{M}_{3}$ on $H$. Then the images of each generator of $P S L_{2}\left(F_{7}\right)$ are given by the following matrices [6]:

$$
\rho(s)=\left(\begin{array}{cccccc}
-1 & 0 & 0 & 0 & 0 & -1 \\
0 & -1 & 0 & 0 & 0 & -1 \\
-1 & -1 & 1 & 1 & 1 & 0 \\
0 & 0 & 0 & -1 & 0 & -1 \\
0 & 0 & 0 & 0 & -1 & -1 \\
0 & 0 & 0 & 0 & 0 & 1
\end{array}\right), \quad \rho(t)=\left(\begin{array}{cccccc}
1 & 0 & 0 & 0 & -1 & 0 \\
1 & 0 & 0 & 0 & 0 & 1 \\
1 & 0 & 0 & 0 & 0 & 0 \\
0 & 1 & 0 & 0 & 0 & 1 \\
1 & 0 & -1 & 0 & -1 & 0 \\
-1 & -1 & 1 & 1 & 1 & 0
\end{array}\right) .
$$

Moreover to compute the $\eta$-invariant we need matrices $\rho([s, t])$ and $\rho(s t)$. Using the above Sp-representations, from the definition we can directly calculate Meyer's cocycle $\tau_{3}$ at level 3 .

As for the definition of the $\eta$-invariant of the signature operator, see the original paper [2]. To be brief, it measures the extent to which the Hirzebruch signature formula fails for a non-closed $4 k$-dimensional Riemannian manifold whose metric is a product near its boundary.

Let $M_{\varphi^{n}}(1 \leq n \leq m)$ be a mapping torus determined by $\varphi \in P S L_{2}\left(F_{7}\right) \subset \mathcal{M}_{3}$, where $m$ is the order of $\varphi$. Namely it is the identification space $S \times[0,1] /(p, 0) \sim$ $\left(\varphi^{n}(p), 1\right)$. Then the $\eta$-invariant of $M_{\varphi^{n}}$ is given by

$$
\eta\left(M_{\varphi^{n}}\right)=-\sum_{k=1}^{n-1} \tau_{3}\left(A, A^{k}\right)+\frac{n}{m} \sum_{k=1}^{m-1} \tau_{3}\left(A, A^{k}\right),
$$

where $A=\rho(\varphi)$ (see [8]). From this formula we can compute the $\eta$-invariant of mapping torus corresponding to each element of $G$.

Proposition 2. For each element of $P S L_{2}\left(F_{7}\right)$, explicit lists of the $\eta$-invariant of mapping tori are given in Table 2. 
TABLE 2. Signature cocycles and $\eta$-invariants for $P S L_{2}\left(F_{7}\right)$

\begin{tabular}{c|c|c}
\hline Order $m$ & $\tau_{3}\left(A, A^{k}\right)(1 \leq k \leq m-1)$ & $\eta\left(M_{\varphi^{n}}\right)(1 \leq n \leq m)$ \\
\hline 1 & 0 & 0 \\
2 & 0 & 0 \\
3 & 0 & 0 \\
4 & 0 & 0 \\
$7_{1}$ & $-2(k=1,5)$ & $-2(n=1,2,4)$ \\
& $-6(k=2,4)$ & $2(n=3,5,6)$ \\
& $2(k=3)$ & $0(n=7)$ \\
& $0(k=6)$ & \\
\hline
\end{tabular}

Remark 3. For the conjugacy classes of order 7 , we present the values of the signature cocycle and the $\eta$-invariant only for the class $7_{1}$, since a representative of $7_{2}$ is given by the inverse of $7_{1}$. Then each value of $7_{1}$ and $7_{2}$ have the opposite sign.

\section{Proof of Theorem 1}

An essential 1-submanifold of $S$ is a disjoint union of simple closed curves in $S$ each component of which does not bound a 2-disk in $S$, and no two components of which are homotopic. An automorphism of $S$ is reducible if it has a representative which leaves some essential 1-submanifold of $S$ invariant.

Proof. For a surface of odd genus $\sigma$, a finite cyclic action on it is reducible if and only if the order of a generator is less than $2 \sigma+1$ [5]. Therefore Theorem 1 immediately follows from Proposition 2.

\section{ACKNOWLEDGMENTS}

The author would like to express his gratitude to Professor Shigeyuki Morita for his encouragement and many useful suggestions. He also would like to thank Dr. Yasushi Kasahara for pointing out this topic and for helpful discussions.

\section{REFERENCES}

[1] M. F. Atiyah, On framings of 3-manifolds, Topology 29 (1990), 1-7. MR 91g:57025

[2] M. F. Atiyah, V. K. Patodi, I. M. Singer, Spectral asymmetry and Riemannian geometry I, Math. Proc. Camb. Phil. Soc. 77 (1975), 43-69. MR 53:1655a

[3] M. Conder, Hurwitz groups: A brief survey, Bull. A.M.S. New Ser. 23 (1990), 359-370. MR 91d:20032

[4] I. M. Isaacs, Character theory of finite groups, Academic Press, New York (1990). CMP 94:14

[5] Y. Kasahara, Reducibility and orders of periodic automorphisms of surfaces, Osaka J. Math. 28 (1991), 985-997. MR 93g:57021

[6] A. Matsuura, The automorphism group of the Klein curve in the mapping class group of genus 3, Proc. Japan Acad. 72 Ser. A (1996), 139-140. CMP 97:04

[7] W. Meyer, Die Signatur von Flächenbündeln, Math. Ann. 201 (1973), 239-264. MR 48:9715

[8] T. Morifuji, The $\eta$-invariant of mapping tori with finite monodromies, Topology Appl. 75 (1997), 41-49. CMP 97:05

Graduate School of Mathematical Sciences, University of Tokyo, Komaba, Meguro, TOKYO 153, JAPAN

E-mail address: morifuji@ms406ss5.ms.u-tokyo.ac.jp 